\title{
Experimental and theoretical evaluation of the trapping performance of polymeric lensed optical fibers: single biological cells versus synthetic structures
}

Joana S. Paiva, Rita S. R. Ribeiro, Pedro A. S. Jorge, Carla C. Rosa, Maria M. Azevedo, et al.

Joana S. Paiva, Rita S. R. Ribeiro, Pedro A. S. Jorge, Carla C. Rosa, Maria M. Azevedo, Paula Sampaio, Joao P. S. Cunha, "Experimental and theoretical evaluation of the trapping performance of polymeric lensed optical fibers: single biological cells versus synthetic structures," Proc. SPIE 10685, Biophotonics: Photonic Solutions for Better Health Care VI, 106852W (17 May 2018); doi: 10.1117/12.2304358 


\title{
Experimental and Theoretical Evaluation of the Trapping Performance of Polymeric Lensed Optical Fibers: Single Biological Cells versus Synthetic Structures
}

\author{
Joana S. Paiva ${ }^{\mathrm{a}, \mathrm{b}}$, Rita S. R. Ribeiro ${ }^{\mathrm{a},{ }^{* *}}$, Pedro A. S. Jorge ${ }^{\mathrm{a}, \mathrm{b}}$, Carla C. Rosa ${ }^{\mathrm{a}, \mathrm{b}}$, Maria M. \\ Azevedo $^{c}$, Paula Sampaio ${ }^{c}$, and João P. S. Cunha ${ }^{\mathrm{a}, \mathrm{d}}$ \\ ${ }^{a}$ INESC Technology and Science, INESC TEC, Portugal \\ ${ }^{b}$ Faculty of Sciences, University of Porto, Physics and Astronomy Department, Portugal \\ ci3S - Instituto de Investigação e Inovação em Saúde \\ ${ }^{\mathrm{d}}$ Faculty of Engineering, University of Porto, Portugal
}

\begin{abstract}
Optical Tweezers (OTs) have been widely applied in Biology, due to their outstanding focusing abilities, which make them able to exert forces on micro-sized particles. The magnitude of such forces (pN) is strong enough to trap their targets. However, the most conventional OT setups are based on complex configurations, being associated with focusing difficulties with biologic samples. Optical Fiber Tweezers (OFTs), which consist in optical fibers with a lens in one of its extremities are valuable alternatives to Conventional Optical Tweezers (COTs). OFTs are flexible, simpler, low-cost and easy to handle. However, its trapping performance when manipulating biological and complex structures remains poorly characterized. In this study, we experimentally characterized the optical trapping of a biological cell found within a culture of rodent glial neuronal cells, using a polymeric lens fabricated through a photo-polymerization method on the top of a fiber. Its trapping performance was compared with two synthetic microspheres (PMMA, polystyrene) and two simple cells (a yeast and a Drosophila Melanogaster cell). Moreover, the experimental results were also compared with theoretical calculations made using a numerical model based on the Finite Differences Time Domain. It was found that, although the mammalian neuronal cell had larger dimensions, the magnitude of forces exerted on it was the lowest among all particles. Our results allowed us to quantify, for the first time, the complexity degree of manipulating such "demanding" cells in comparison with known targets. Thus, they can provide valuable insights about the influence of particle parameters such as size, refractive index, homogeneity degree and nature (biologic, synthetic). Furthermore, the theoretical results matched the experimental ones which validates the proposed model.
\end{abstract}

Keywords: Optical trapping, Optical Fiber Tweezers, Micromanipulation, Drag Force Method, Finite Difference Time Domain Method (FDTD), Cell Manipulation

\section{INTRODUCTION}

Optical Tweezers (OTs) have been widely used in Biology-related fields. ${ }^{1,2}$ Due to their ability to exert piconewton forces on microparticles, they can be used to study the mechanical behaviour of cells or to manipulate organelles inside cells. ${ }^{3-5}$ These tools are typically characterized by outstanding light focusing abilities. OT trapping forces result from the momentum transfer between the radiation field that is tightly projected onto the scatterer and this latter. The resultant energy is then enough to trap the target in a contactless and minimally invasive manner. ${ }^{4-6}$ Two components characterize this trapping force: the dipole and gradient forces. The first one results from the electromagnetic field inhomogeneities and spatial intensity gradients and is responsible for pulling the scatterer towards the highest intensity region. On the other hand, the scattering force is proportional to the imaginary part of the complex target polarizability, and pushes the particle along the incident light

Further author information: (Send correspondence to J.S.P.)

J.S.P.: E-mail: jipaiva@inesctec.pt

${ }^{* *}$ Rita S. R. Ribeiro is currently with with 4Dcell and Elvesys, Paris, France. 
propagation direction. A given particle is therefore stably trapped when the gradient force counterbalances the scattering force of the beam intensity steep spatial gradient, and the resultant of forces applied to the particle is null. ${ }^{5}$

There are several OT setups proposed in literature, ranging from simple configurations, lens-based traps to complex instrumentation systems. ${ }^{3,4,6-8}$ The most conventional OT setups - the Conventional Optical Tweezers (COTs) - are usually composed by a laser, a set of optical components to expand and steer the light beam, a high numerical aperture objective, an observation system and a sample holder, all packaged together in a form of an inverted microscope. ${ }^{3}$ Despite being broadly used, these setups are associated with several drawbacks, including small working distances, limited portability and high complexity degrees, focusing difficulties when handling turbid media or thick samples as biologic preparations. They are also expensive and have low flexibility degrees. $^{8-10}$ Optical Fiber Tweezers (OFTs), which consist in optical fibers with the extremity patterned or modified to form a lens to tightly focus the propagating laser beam have been considered a valuable alternative to COTs. ${ }^{1}$ OFTs are flexible, simpler and easy to handle. In fact, by changing the chemical, geometric and/or optical properties of the tip of optical fibers it is possible to obtain tools that, beyond guiding light or measuring variables, are able to trap and manipulate particles. ${ }^{3,8-10}$ This kind of platforms can be in future extremely valuable to understand how to intercede in several biocellular phenomena at the microscale (e.g., neuronal traffic, axonal growth), for being flexible and biocompatible and able to reach remote locations. ${ }^{11}$

Despite of the advantages of OFTs, the range of targets manipulated by these newly developed tools is still limited, as well as their mechanical and biological effects on more complex cellular structures remain poorly understood. ${ }^{10,12,13}$ Only synthetic particles or simple biologic cells (e.g., yeasts, bacteria, Red Blood Cells) were so far successfully trapped using OFTs. ${ }^{4,9,14,15}$ To the best of our knowledge, the most complex cellular entity that was manipulated by such kind of optical tools were neuroblastoma cells derived from a rodent cellular culture. ${ }^{16}$ However, how demanding the trapping of such complex cells in comparison with reference targets as synthetic particles, and which trapping lens characteristics influence the most trapping stability of complex biologic targets are aspects which, even theoretically, are not yet well understood.

In order to experimentally and theoretically evaluate how difficult could be to trap a rodent neural-derived cellular structure in comparison with synthetic targets, we conducted a study in which the trapping of a complex biological cell found within a culture of rodent glial cells by a polymeric lensed fiber was characterized. The optical forces exerted by a polymeric lens fabricated through a low-cost photo-polymerization method on this "biostructure" were determined through the Drag Force method and compared with the profile of forces exerted by the same lens and laser power value on three types of simple particles: two $4 \mu \mathrm{m}$-radius spheres (one of PMMA and other of polystyrene) and a $3.5 \mu \mathrm{m}$-radius yeast cell. Trapping forces profile differences were also evaluated considering the mammalian structure and a Drosophila Melanogaster cell. The latter, despite not being as simple as a yeast cell, allowed to compare the mammalian structure with a similar cell in terms of size, but still different enough in complexity. Moreover, the feasibility and reliability of a numerical model for trapping forces calculation previously proposed by our $l a b^{3,9}$ was also evaluated considering complex structures as the one manipulated in the experiments conducted.

Our results allowed to quantify how hard can be the manipulation of complex cells versus known targets through optical trapping. They can provide valuable insights about the real influence of particle parameters such as size, refractive index, composition homogeneity degree and nature (biologic, synthetic) on trapping. Additionally, the proposed numerical model have adequately predicted experimental results, which confirms its validity in describing the trapping of complex structures. Both experimental and theoretical conclusions can be important to understand in which optical and experimental conditions such "demanding" biological structures as the mammalian ones or even simpler cells as Drosophila Melanogaster cells could be successfully trapped using simple and flexible OFT configurations, having potential implications on future OFT-based micro and nano particles sensing devices. ${ }^{2}$

\section{METHODS}

\subsection{Optical Trapping Experiments}

In the following subsections, the fabrication method of the polymeric lenses on top of optical fibers, as well as the custom-build inverted microscope setup used for particles manipulation and visualization - see fig.1 - will be 
described. The optical fiber selected to work with was a $980 \mathrm{~nm}$ Thorlabs single mode fiber (SMF) (Thorlabs SM 980-5.8-125).

\subsubsection{Polymeric Fiber Tip Fabrication Method}

The polymeric trapping lens used in this study was fabricated using a guided photo-polymerization method that was developed by Soppera et $\mathrm{al}^{17}$ in collaboration with our laboratory. ${ }^{3,18-21}$ Polymeric OFTs fabricated using this process and able to successfully trap in 2D both PMMA and polystyrene beads, yeast cells and algae were already obtained in our laboratory. ${ }^{3,9,17-21}$ Through this process, it is possible to obtain a spherical lens on the top of an optical fiber, which results from the assemble of cross-linked polymeric microstructures, triggered by light of a specific wavelength. ${ }^{3,18-21}$ For the photo-polymerization process, the monomer pentaerythriol triacrylate (PETIA) - $n=1.48$ - and the photo-initiator Bis(2,4,6-trimethylbenzoyl)-phenylphosphineoxide, commercially known as Irgacure 819 , were used in a polymer mixture containing $0.2 \%$ of photo-initiator for the quantity of monomer. Since the Irgacure 819 is sensitive to wavelength values between 375 and $450 \mathrm{~nm}$, a violet diode $405 \mathrm{~nm}$ laser (LuxX cw, $60 \mathrm{~mW}$, Omicron) was used to trigger the polymeric cross-linking chain reaction. ${ }^{3}$ This fabrication method can be summarized by the following steps: at first, an optical fiber is cleaved at one of its extremities and is positioned vertically in a moving stage, while the laser is aligned to be injected in its distal end and to excite the fundamental mode. Then, the cleaved end of the optical fiber is dropped into the polymer solution, aided by the moving stage. After being removed from this solution, the polymer drop formed in fiber extremity is cured by a laser power of at least $20 \mu \mathrm{W}$ at $405 \mathrm{~nm}$, during 60 seconds. Then, the remaining non-polymerized solution was washed out from the cured tip using ethanol. This method is characterized by a self-guiding effect since during polymerization the refractive index of the growing tip increases, which creates a waveguide effect. The visual aspect of the fabricated polymeric lens is provided in fig. 2.(A). It is important to mention that the optical fiber used in this experiment is multimode at a wavelength of $405 \mathrm{~nm}$ and the diameter of the lenses fabricated through this process usually matches the fiber modal diameter corresponding to the guided mode that is excited during the irradiation process. Thus, since low order modes yield more smooth tips, ${ }^{3,18-21}$ an effort was made to excite always the fundamental one.

\subsubsection{Particles Manipulation Setup}

A custom-build inverted microscope setup was developed for trapping and manipulating microspheres using the fabricated optical fiber tip through the process described above - please see fig. 1 for a scheme of this setup. It was composed by a 20x objective connected to an image acquisition and a 4-axis motorized micromanipulator ( $x, y, z$ and angular) to handle the optical fiber. A pigtailed $980 \mathrm{~nm}$ laser (500 $\mathrm{mW}$, Lumics, ref. LU0980M500) was connected to the fabricated tip which was then inserted into a metallic capillary carefully controlled by the motorized micromanipulator. The capillary was tilted at $50^{\circ}$, since trapping phenomena is only possible for fiber tip inclination angles $>30^{\circ} .{ }^{9}$ The image acquisition system was composed by a CMOS camera (EO-2018C, Edmund Optics) connected to a laptop. A drop of dPBS containing the microparticles/cells - from solutions 1 to 5 (table 1) - was then placed over a glass coverslip over the inverted microscope setup, and the optical fiber tip was immersed on it. In this experiment, five types of particles/cells were analyzed: two synthetic particles (two $8 \mu \mathrm{m}$ diameter PS and PMMA microspheres), two simple biological cells (a $7 \mu \mathrm{m}$ diameter yeast and a $12.5 \mu \mathrm{m}$ diameter Drosophila Melanogaster cell), and a more complex biologic entity (a cell found in a culture of rodent glial cells with an oval geometry - $15 \mu \mathrm{m}$ major axis; $10.5 \mu \mathrm{m}$ minor axis) - please consult table 1 . The immersed micro tip as well as the manipulated microparticles were visualized using the CMOS camera during trapping experiments. The output trapping laser power was set to $\approx 158 \mathrm{~mW}$ (@980 nm) for all the manipulated particles (PMMA, PS microspheres; yeast cells, glial structure and Drosophila Melanogaster cell). Figs. 2.(B) and 2.(C) show the yeast cell and the mammalian structure trapped, respectively, by the polymeric lensed fiber. Optical trapping forces were calculated through the Drag Force method ${ }^{22}$ - please read point 2.1.3.

\subsubsection{Trapping Forces Calculation - Drag Force method}

The method employed in this study to measure optical trapping forces is based on the Faxen's law, a revised form of the Stokes equation. ${ }^{22,27}$ This altered version of the drag force equation considers that the trapped particle is close to a boundary (in this particular case, the glass slab). Thus, the total optical force exerted on the particle is given by the sum of the inertial force and the drag force: 




Figure 1. Optical manipulation setup.
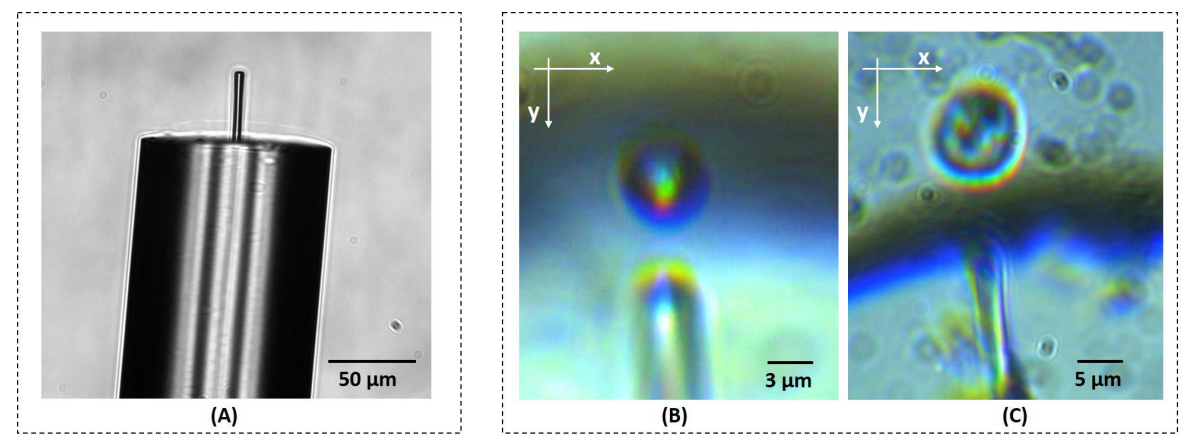

Figure 2. Trapping of microstructures suspended in dPBS (Dulbecco's phosphate-buffered saline) using the fabricated polymeric lens on the top of a fiber. (A) Microscopic image (obtained using the Zeiss Axiovert 200M inverted microscope) of a polymeric optical fiber tip fabricated using the photo-polymerization method described. (B and C) Polymeric optical lens trapping (B) a yeast cell and (C) a mammalian cellular microstructure suspended in dPBS - images acquired using the custom-built inverted microscope setup described above.

$$
F_{T}=F_{\text {inertial }}+F_{\text {drag }}=m \frac{\partial^{2} s}{\partial t^{2}}+6 \pi \xi \eta r \frac{\partial s}{\partial t},
$$

in which $m$ is the mass of the trapped particle, $s(t)$ represents the particles trajectory during manipulation, $\xi$ a correction factor due to the proximity to the trapping chamber (in this particular case, $\xi=3.08$ ) ${ }^{9} \eta$ the viscosity of the media (in this case, dPBS, $9.04 \times 10^{-4} \mathrm{~Pa}$ ) and $r$ is the radius of the particle. Considering the low Reynolds number associated with this particular situation $\left(R e \approx 10^{-59}\right)$, the inertial force is negligible. Thus, in trapping conditions there is a balance between the optical trapping force and the drag force. The trapping force $\left(F_{T}\right)$ acting on the particle can then be calculated according to the following:

$$
F_{T}=6 \pi \xi \eta r \frac{\partial s}{\partial t}
$$

Once the viscosity of the media $\eta$, the radius of the particle $r$, the correction factor $\xi$ are known variables, it is only necessary to calculate the velocity of the particle $\left(\frac{\partial s}{\partial t}\right)$ when manipulated to calculate trapping forces (please consult equation defined in 2).

To experimentally determine the optical force exerted on each particle considered, the following sequence of events were recorded using the CMOS camera. After the particle being stably trapped in front of the polymeric tip as depicted in figs. 2.(B) and 2.(C), the laser was turned off and the tip was moved by few micrometers away from the particle. Then, the laser was again turned on and the particle was attracted towards the stable 
trapping position, while its movement was recorded using the CMOS camera, controlled by the uEye Cockpit software from $\operatorname{IDS}^{\circledR}$, at a sampling rate of 50 frames per second. Due to the cellular complexity degree of the glial structure, its displacement along the longitudinal direction (up and down movements, $-y$ and $+y$ ) was almost insignificant. For that reason, only transversal forces were studied. Thus, particles trajectories were monitored for both directions $-x$ and $+x$ (towards the left and right directions, respectively), using a laser power of $158 \mathrm{~mW}$ (@980 nm) with the fiber titled at $50^{\circ}$. Particles displacement events due to optical trapping were repeated for four times for each direction $(-x$ and $+x)$ and each particle, to obtain a statistical profile of the measurements.

After particles trajectory video acquisition, frames were imported to MATLAB R2015a and trajectories were tracked using the open-source MATLAB compatible software CellTracker. ${ }^{28}$ Then, particles position for each time point data was fit to the Langevin equation, ${ }^{29}$ in order to obtain a smoother curve for particles trajectory description. ${ }^{9}$ Thus, the velocity of particles during the restoring movement (first derivative of particles position versus time) was possible to obtain for each direction $(-x$ and $+x)$ and each particle type. Trapping forces profile corresponding to each condition was then determined by the equation defined in 2 and compared between the different particles.

Optical trapping forces were experimentally compared between four particle types: synthetic ( $8 \mu \mathrm{m}$ diameter polystyrene and PMMA microspheres), one biologic with low complexity degree (yeast cell), and a complex mammalian structure from a rodent primary glial cell culture. Considering the size difference between the yeast and the glial structure, we also compared trapping force profiles between the glial and a Drosophila Melanogaster round cell whose complexity degree is slightly higher than yeasts, but still highly limited in terms of genetic information complexity. Please consult table 1 for a summary of the characteristics of each particle.

\subsection{Computational Model and Simulations}

In order to investigate the validity and feasibility of a possible theoretical model to mathematically explain trapping forces of complex cells using polymeric lenses, we compared the above experimental results with theoretical simulations obtained using a previously proposed model. ${ }^{3,8,9,30}$ This is based on the work developed by Barnett and Loudon and has already showed to be suitable for modeling the trapping phenomena of simple, synthetic and perfectly rounded particles. ${ }^{3,9}$

\subsubsection{Trapping Forces Calculation}

According to the model of Barnett and Loudon, ${ }^{31}$ the optical force exerted on each particle can be modeled as a Lorentz force exerted on the microscopic dipoles which compose the particle. In our theoretical simulations, optical forces computation was performed in two steps, and simulations parameters were set to match, as closer

Table 1. Optical and morphological characteristics of the particle samples used in the experiment. dPBS - Dulbecco's phosphate-buffered saline. RI - Refractive Index.

\begin{tabular}{|c|c|c|c|c|c|}
\hline & & Media & Particle Type & $\begin{array}{c}\text { Trapped Particle } \\
\text { Dimensions }\end{array}$ & Particles RI \\
\hline \multirow{3}{*}{ Synthetic and simple biological structures } & Solution 1 & \multirow{5}{*}{$\mathrm{dPBS}(\mathrm{n}=1.36)$} & $\begin{array}{l}\text { Polystyrene } \\
\text { microspheres }\end{array}$ & $8 \mu \mathrm{m}$ diameter & $1.580^{23}$ \\
\hline & Solution 2 & & $\begin{array}{c}\text { PMMA } \\
\text { microspheres }\end{array}$ & $8 \mu \mathrm{m}$ diameter & $1.480^{23}$ \\
\hline & Solution 3 & & Yeast cells & $7 \mu \mathrm{m}$ diameter & $1.500^{24}$ \\
\hline \multirow[t]{2}{*}{ More complex biological structures } & Solution 4 & & $\begin{array}{c}\text { Rodent glial } \\
\text { neuronal cells and } \\
\text { structures }\end{array}$ & $\begin{array}{c}10.5 \mu \mathrm{m} \text { minor } \\
\text { axis, } 15 \mu \mathrm{m} \text { major } \\
\text { axis }\end{array}$ & $1.380^{25}$ \\
\hline & Solution 5 & & $\begin{array}{c}\text { Drosophila } \\
\text { Melanogaster cells }\end{array}$ & $12.5 \mu \mathrm{m}$ diameter & $1.365^{26}$ \\
\hline
\end{tabular}


Table 2. Simulation parameters used in our theoretical model.

\begin{tabular}{|c|c|c|c|}
\hline \multicolumn{4}{|c|}{ Simulation Parameters } \\
\hline \multirow{4}{*}{ Optical System } & Computational Grid & $\begin{array}{l}\text { Dimensions (length } \mathrm{x} \text { width) } \\
\text { Spatial resolution (length } \mathrm{x} \text { width) }\end{array}$ & $\begin{array}{c}90 \mu \mathrm{m} \times 36 \mu \mathrm{m} \\
0.053 \mu \mathrm{m} \times 0.057 \mu \mathrm{m}\end{array}$ \\
\hline & Waveguide & $\begin{array}{c}\text { Curvature radius } \\
\text { RI }\end{array}$ & $\begin{array}{l}3 \mu \mathrm{m} \\
1.458\end{array}$ \\
\hline & Source & $\begin{array}{l}\text { Wavelength } \\
\text { Duration }\end{array}$ & $\begin{array}{c}980 \mathrm{~nm} \\
\text { Continuous }\end{array}$ \\
\hline & Media (dPBS) & RI & 1.360 \\
\hline \multirow{4}{*}{ Targets } & PMMA & $\begin{array}{c}\text { Shape and Dimensions } \\
\text { RI }\end{array}$ & $\begin{array}{c}\text { Rounded, } 8 \mu \mathrm{m} \text { diameter } \\
1.480\end{array}$ \\
\hline & Polystyrene & $\begin{array}{l}\text { Shape and Dimensions } \\
\text { RI }\end{array}$ & $\begin{array}{c}\text { Rounded, } 8 \mu \mathrm{m} \text { diameter } \\
1.580\end{array}$ \\
\hline & Yeast & $\begin{array}{c}\text { Shape and Dimensions } \\
\text { RI }\end{array}$ & $\begin{array}{c}\text { Rounded, } 7 \mu \mathrm{m} \text { diameter } \\
1.500\end{array}$ \\
\hline & Glial structure & $\begin{array}{l}\text { Shape and Dimensions } \\
\text { RI }\end{array}$ & $\begin{array}{c}\text { Oval; } 15 \mu \mathrm{m} \text { major and } 10.5 \mu \mathrm{m} \text { minor axes } \\
1.380\end{array}$ \\
\hline
\end{tabular}

as possible, the real experimental conditions. At first, the stationary electromagnetic field was propagated in two dimensions considering a simulation environment composed by a 90x36 $\mu \mathrm{m}$ computational grid (see fig. 3), a waveguide with the same characteristics as our polymeric tip and the target (each one of the particle considered) located in different positions in the grid - please consult table 2, in which all the simulations parameters values used are listed. Field propagation was performed using the Finite Differences Time Domain (FDTD) method implemented on the MEEP software ${ }^{32}$ compatible with Python $^{\text {TM }}$. Then, after propagating the electromagnetic field till stabilizing, optical trapping forces can be computed, for each particle position considered, by integrating over the particle the force per grid position $i$, defined by the equation:

$$
f_{i}=\frac{1}{4} \cdot \epsilon_{0} \cdot\left(\epsilon_{p}-\epsilon_{m}\right) \Delta_{i}\left|E^{2}\right|,
$$

in which $\epsilon_{0}$ is the vacuum permittivity, $\epsilon_{p}$ is the particle relative permittivity and $\epsilon_{m}$ is the media relative permittivity. ${ }^{8-10,31}$ Theoretical trapping forces were computed for different 510 positions relatively to the waveguide, for the four particles described in table 2 and compared with the experimental results obtained. All theoretical simulations were computed using custom-built MATLAB ${ }^{\circledR}$ and Python ${ }^{\text {TM }}$ scripts. In fig. 3 is provided a graphical representation of the simulation environment considered (including the waveguide and a particle positioned in one of the 510 positions considered).

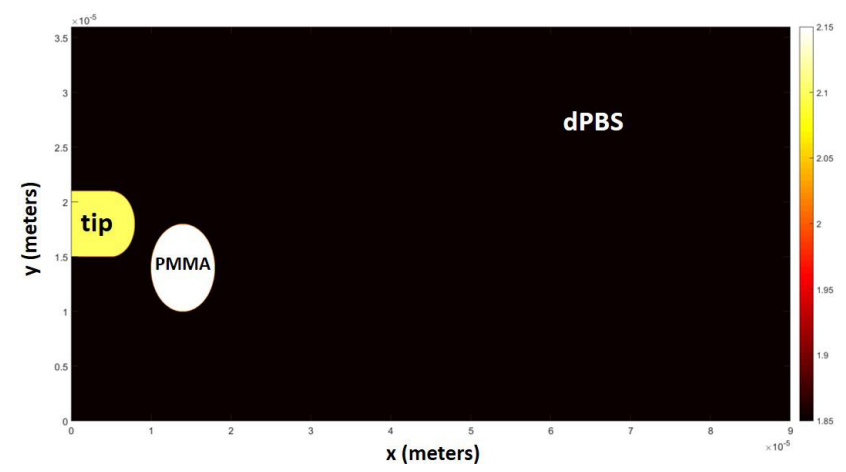

(A)

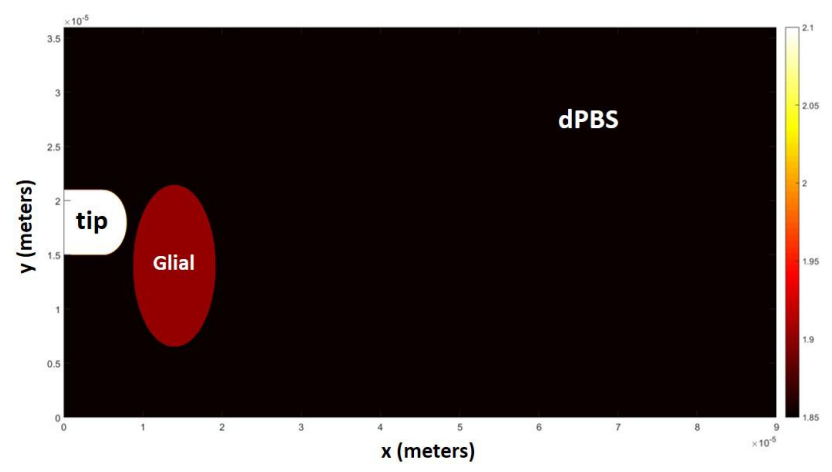

(B)

Figure 3. Permittivity 2D map of the optical simulation system including (A) the PMMA microsphere as target and (B) the mammalian glial structure as scatterer. Colored bar at right represents permittivity values correspondence. 


\section{RESULTS AND DISCUSSION}

\subsection{Trapping Experiments}

\subsubsection{Optical Trapping}

As previously reported in studies conducted by our lab, ${ }^{3,9,18,19,33}$ the polymeric spherical lenses fabricated through the guided photo-polymerization method described in section 2.1.1 are able to stably trap and manipulate all the particles selected for this study ( $8 \mu \mathrm{m}$ diameter PMMA and Polystyrene microspheres, yeast cells, glial mammalian structure and Drosophila Melanogaster cells). Sequences of video snapshots of particles trapping by the lensed optical fiber tip are provided in fig. 4. Each sequence represents how trapping forces were calculated through the Drag Force method. All the images numbered with (I), (III) and (V) for particles (A-E) represent when the optical fiber tip was displaced relatively to its original position with the laser turned off. Images (II), (IV) and (VI) from (A-E) represent particle trajectory when the laser was turned on and the particle was attracted to the trapping equilibrium position in front of the fiber, for each particles type. Synthetic particles (from PMMA and Polystyrene), yeast and Drosophila Melanogaster cells were successfully trapped both in transversal and longitudinal directions. Displacements along the longitudinal direction (up and down movements relative to the optical fiber tip) were almost insignificant for the glial structure. For this reason, only the transversal profile of trapping forces was compared between particles (corresponding to scatterers displacement towards to the left $(-x)$ and right $(+x)$ directions) - please read sections 3.1.2 and 3.1.3.

\subsubsection{Forces profile for synthetic and biological structures}

In fig. 5 are depicted examples of trajectories made by each type of particle due to optical trapping using the same polymeric lens and laser power towards the left (A, C, E and $\mathrm{G})$ and right $(\mathrm{B}, \mathrm{D}, \mathrm{F}$ and $\mathrm{H})$ directions, and corresponding fit line obtained through the Langevin approximation to the experimental data. It is possible to observe that, using the same laser power, polystyrene, PMMA microspheres and yeast cell show similar trajectories along time. For these particles, trapping restoring movements were observed at transversal distances of 4-6 $\mu \mathrm{m}$ away from the laser beam propagation direction (figs. 5.(A, B, C, E and F)) - towards both $-x$ and $+x$ directions. This means that, all these particles, when in the boundary of the polymeric lens at a transversal distance equal or less than 4-6 $\mu \mathrm{m}$, were attracted to the trapping equilibrium position in the front of the tip. The time period corresponding to the trapping restoring movement was approximately in the range of 0.3 and 1 seconds for these three types of targets (PS, PMMA spheres and yeast). The glial structure showed a completely different behaviour. Considering the same manipulation tool and trapping power, it was displaced by approximately 14-16 $\mu \mathrm{m}$ due to optical trapping effects. However, its movement was slower than the other three particles (synthetic microspheres and yeast cell). Trapping restoring movement took between 3 and 5 seconds. These values suggest a completely different mechanical reaction of such complex structure to the trapping force, in comparison with the simple scatterers, most likely due to the different geometry, RI, complexity degree and size. However, more attention should be given to the transversal force values in function of the distance to trapping equilibrium position during restoring movements, which graphical representation can be found in fig. 6 .

Considering the force profiles depicted in fig. 6, the restoring movement of particles after the laser being turned on is clearly visible through the curves shape. Theoretically, these graphics should be symmetric relatively to the axes origin and the maximal forces exerted on particles should be of the same magnitude independently of the direction of the movement (right or left). For all particles (A-D), the maximal trapping force magnitude relative to particles movement towards $-x$ direction was higher than for the opposite direction, being the particles trajectory asymmetrical. Probably, considering that this detail was observed for all particle types, this asymmetry is associated with the geometry of the lens. The asymmetry degree of mammalian structure trajectory is higher in comparison with the other particles probably due to its eccentric geometry and complexity. It is important to note that, this structure is asymmetric in terms of shape due to its nature and origin. A comparison of the maximal force magnitude for each movement direction (left or right) exerted on each type of particles is provided in fig. 7. Although the mammalian cell was the largest particle manipulated, the magnitude of the trapping force that was possible to exert on it was the lowest from all the particles analyzed at this point (PMMA and PS microspheres and yeast) for both directions. It was of of $-25.32 \pm 6.84 \mathrm{pN}$ and $14.30 \pm 2.61 \mathrm{pN}$ for left and right directions, respectively; $21.20 \%, 12.90 \%$ and $7.53 \%$ (left); and $42.20 \%, 24.50 \%$ and $24.60 \%$ (right) smaller in comparison with the polystyrene, yeast cell and PMMA, respectively. This was transduced in a limited trapping 

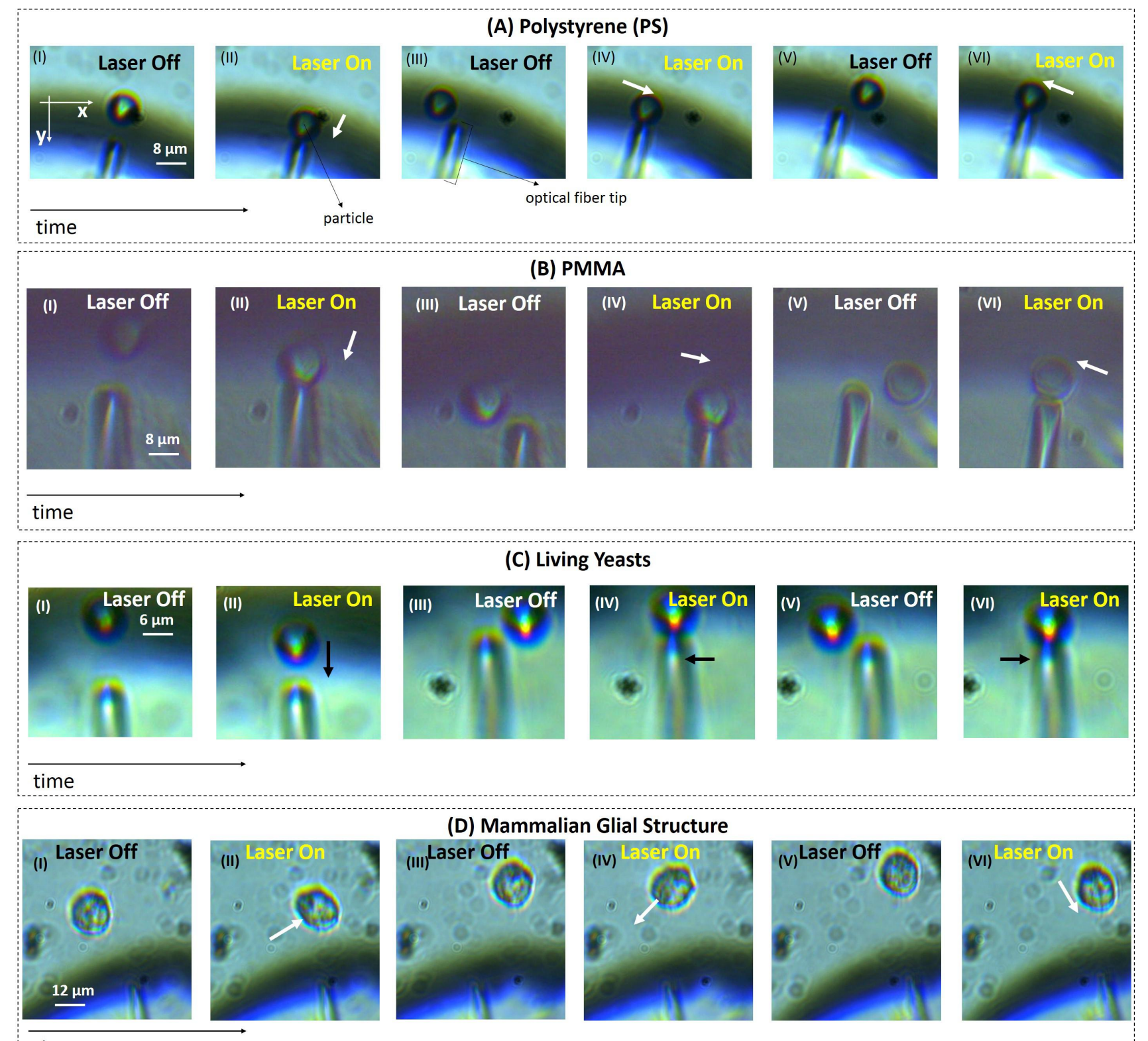

(D) Mammalian Glial Structure


time

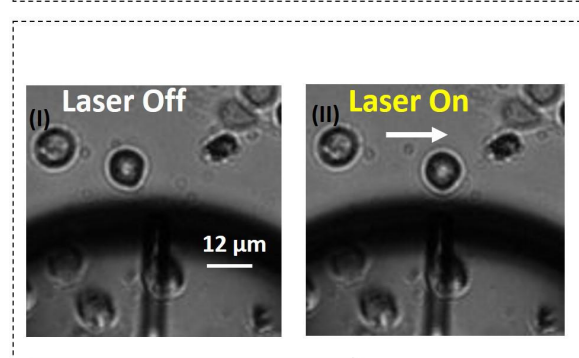

(E) Drosophila Melanogaster cells
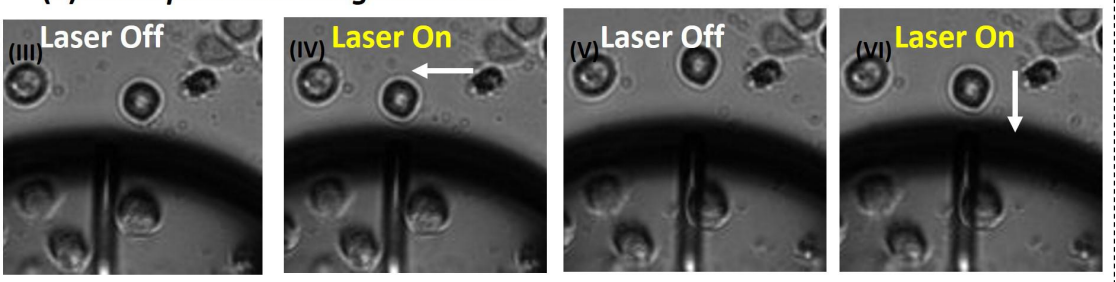

time

Figure 4. Snapshots showing optical trapping of (A) a Polystyrene microsphere, (B) a PMMA microsphere, (C) a yeast cell, (D) the mammalian glial structure and (E) a Drosophila Melanogaster cell due to the displacement of the fabricated polymeric lens. For all the particles (A-D): frames (I), (III) and (V) represent the displacement of fiber tip while the laser is off; and frames (II), (IV) and (VI) show the particle optical trapping phenomena observed after the laser being turned on (the particle is attracted to the trapping point, located in front of the previously displaced fiber tip). The white and black arrows represent the particle movement direction, after the laser being turned on. 


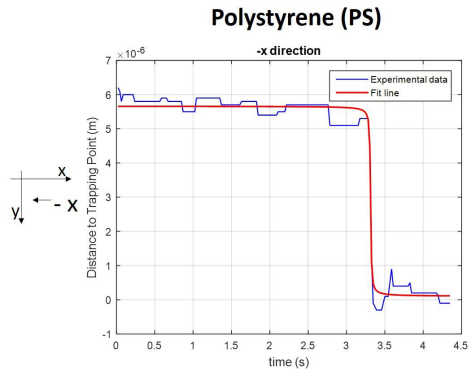

(A)

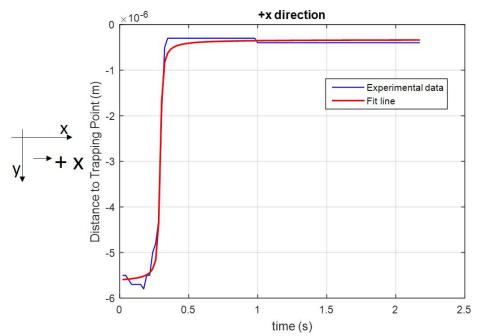

(B)

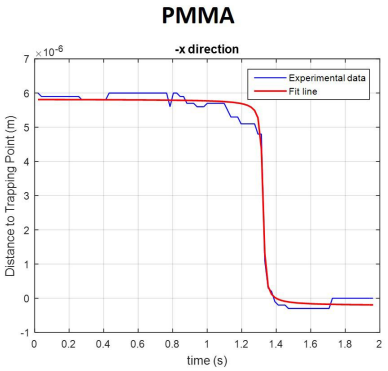

(C)

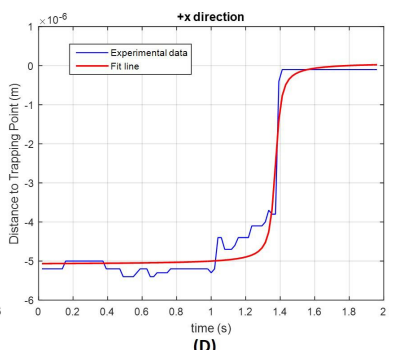

(D)

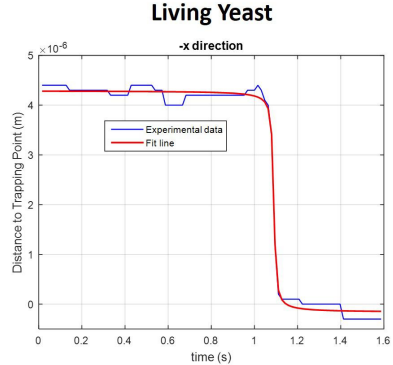

(E)

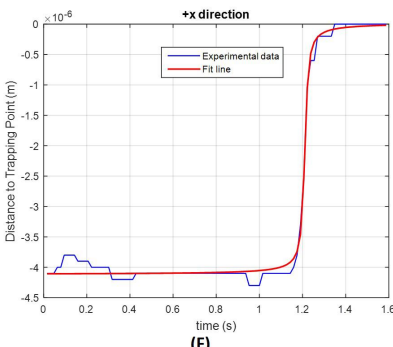

(F)

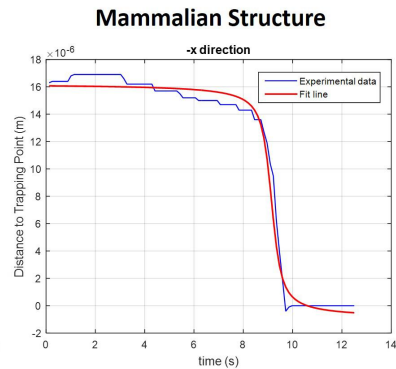

(G)

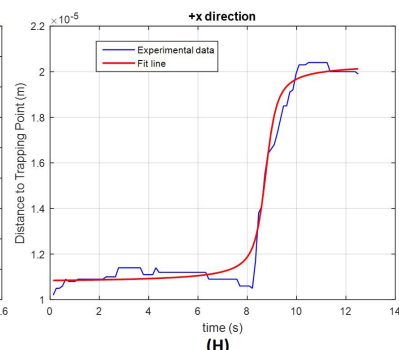

(H)

Figure 5. Example of particles trajectories during optical trapping experiments for the (A, B) polystyrene microsphere; (C, D) PMMA particle; (E, F) yeast cell and $(\mathrm{G}, \mathrm{H})$ glial structure; and corresponding Langevin approximation fit line. $(\mathrm{A}),(\mathrm{C}),(\mathrm{E})$ and $(\mathrm{G})$ represent particles trajectories towards -x direction (when the tip was displaced to the left and the particles were moved into that direction when the laser was turned on); and (B), (D), (F) and (H) are relative to particles trajectories to the right (towards $+\mathrm{x}$ direction) - when the fiber tip was moved to the right and the laser was subsequently turned on.

performance of the biologic cell, on which, for the same laser power, a lower trapping force magnitude was exerted comparing with the other three particles. According to equation 2, the trapping force exerted on a given particle is supposed to increase with the radius of the particle. However, the opposite was observed. The reduced force magnitude that reached the cell can be explained by its reduced refractive index and composition homogeneity degree and high structural complexity order in comparison with the other particles. Additionally, considering that equation 2 does not entirely describe the relation between scattering and gradient forces with the particle radius $(r)$, we must to consider that, probably, a non-balanced relation between the two force components with the particle size can explain the weak trapping phenomena of the mammalian structure. In fact, according to previous studies, ${ }^{9}$ the scattering force depends by a factor of $r^{6}$, while the gradient force increases by a factor of $r^{3}$. Thus, when the particle size increases, the scattering force increases in accordance with the third power of the gradient force, which leads to unstable trapping conditions.

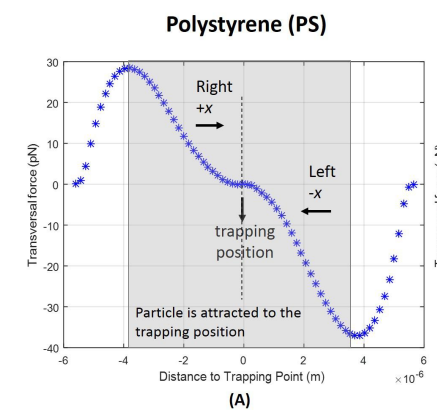

(A)





(C)
Mammalian Structure

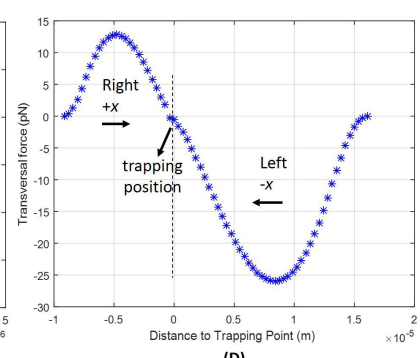

Figure 6. Transversal optical trapping forces profile exerted by the fabricated polymeric lens on (A) the polystyrene particle, (B) the PMMA microsphere, (C) the yeast cell and (D) the mammalian glial structure. 


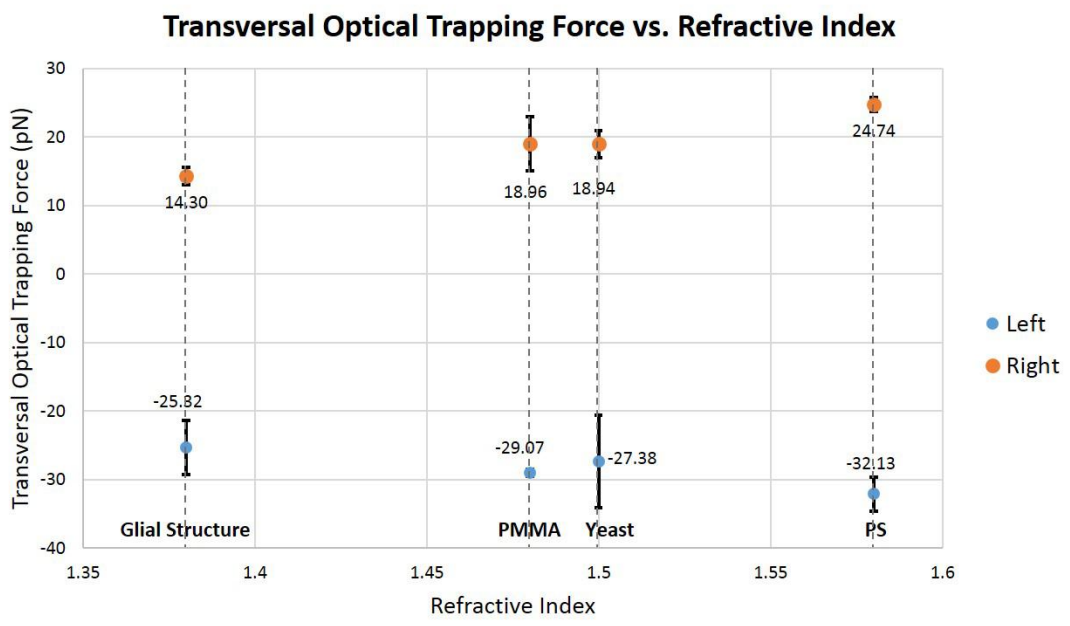

Figure 7. Maximum magnitude of transversal trapping forces for glial neuronal structure, PMMA particle, yeast cell and PS particle exerted by the fabricated polymeric tip. Blue dots represent values relative to particles and fiber tip displacements towards $-\mathrm{x}$ direction (left). Orange dots represent displacements to the $+\mathrm{x}$ direction (right). Error bars represent standard errors.

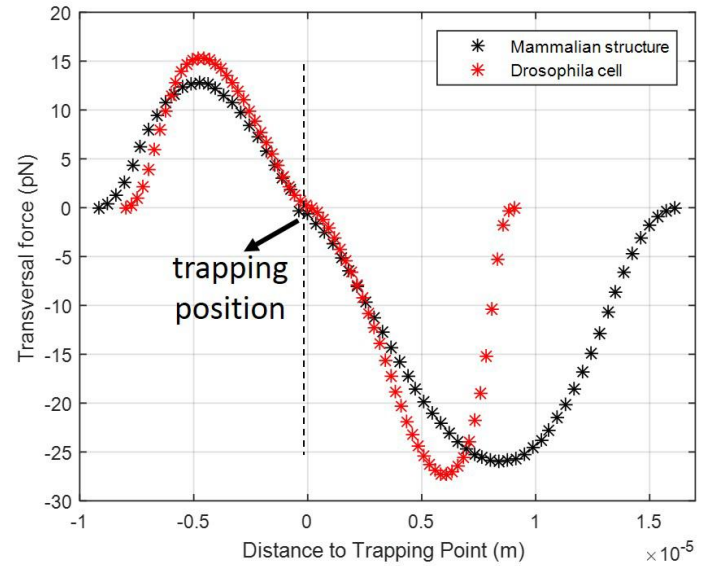

(A)

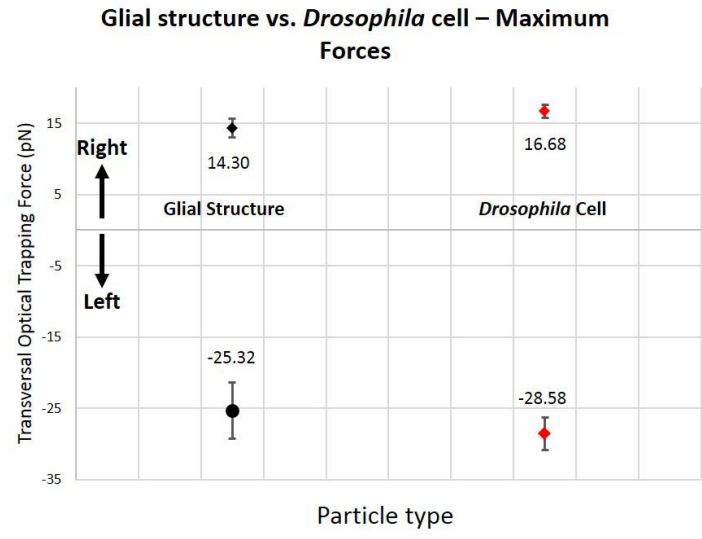

(B)

Figure 8. Trapping force differences between the mammalian glial structure and the Drosophila Melanogaster cell. (A) Tranversal trapping force profile. (B) Maximal forces exerted on both cells when attracted towards the left and right direction due to optical trapping.

\subsubsection{Optical trapping forces comparison between simple and complex biological structures}

Considering that the yeast cell, despite biologic, has much smaller dimensions in comparison with the mammalian structure, we compared the profile of trapping forces exerted on a Drosophila Melanogaster cell, which has low cellular complexity as yeasts and similar dimensions $(12.5 \mu \mathrm{m}$ of diameter) than the mammalian structure, with the trapping forces exerted on the latter. As expected, the maximal magnitude of exerted trapping forces for both displacements towards the left and right direction ( $-x$ and $+x$, respectively) was higher for Drosophila cell than the mammalian cell (see fig. 8). Taking into account that both cells have a similar $2 \mathrm{D}$ area $\left(\approx 491 \mu \mathrm{m}^{2}\right.$ for mammalian cell and $\approx 495 \mu \mathrm{m}^{2}$ for Drosophila cell) and the laser power and polymeric lens used to trap both were always the same, the distinct heterogeneity and complexity degrees between the two structures should be the main reason for such discrepancies in trapping forces magnitude. 


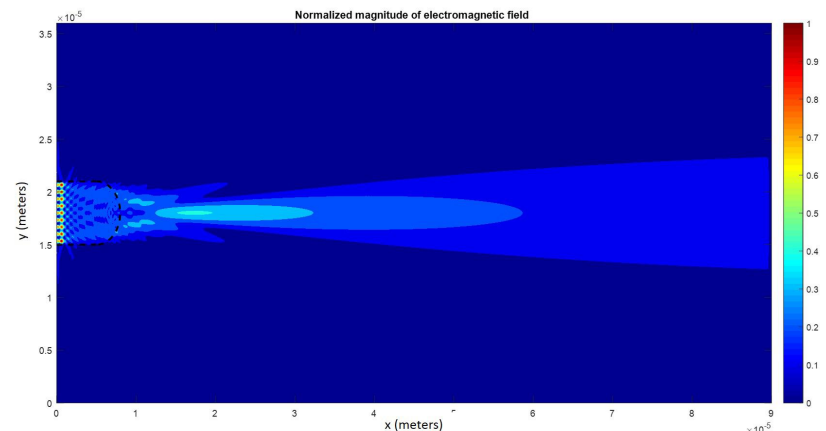

(A)

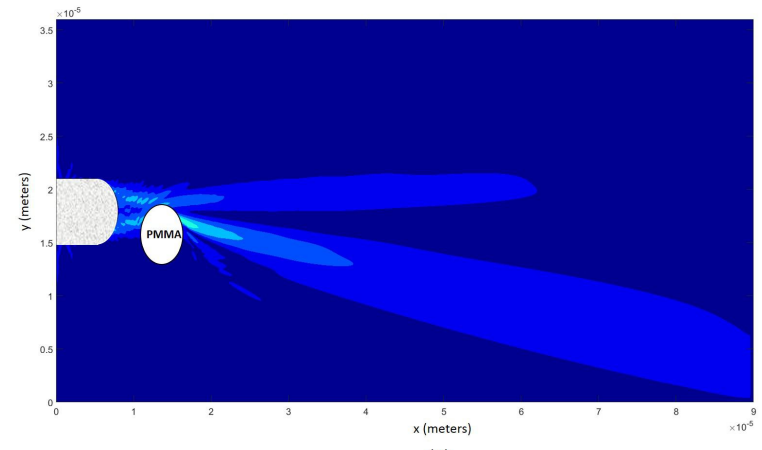

(B)

Figure 9. Electromagnetic field 2D propagation (A) without scatterer and (B) considering a PMMA particle as target.

\subsection{Computational Simulations}

In fig. 9 is depicted a 2D scheme of the electromagnetic field propagation obtained through theoretical simulations (without targets and considering a PMMA microsphere as target). According to theoretical results, the focus of the propagated light beam is located at a distance of $7.4 \mu \mathrm{m}$ from the fiber tip. In the experiments above, for example, yeasts were trapped at a transversal distance of about 6-7 $\mu \mathrm{m}$ from the fiber tip, a value that is approximate to the location of the beam focus according to the theoretical results.

The 2D distribution maps of trapping forces for each particle position are depicted in fig. 10. Considering each distribution map, each vector represents the resultant force exerted on the particle if located in the corresponding spot. For the four particle types, trapping is theoretically possible, as experimentally validated (particles are attracted to the optical axis if within the boundaries delimited by the lines formed by the vectors in red). According to simulations, trapping occurs at a transverse distance of $13.5 \mu \mathrm{m}, 12 \mu \mathrm{m}$, and $6 \mu \mathrm{m}$ for PS/PMMA microspheres, yeast and mammalian structure, respectively (where the resultant of trapping forces exerted on particle is almost null). As in experiments, theoretical results also show that trapping is only possible at two dimensions, considering these conditions. Additionally, observing fig. 10.(D), the longitudinal component of the resultant force is almost insignificant in comparison with the transversal component for the mammalian structure. In fact, up and down displacements of the particle relatively to the fiber tip due to optical trapping were almost imperceptible.

In order to verify the reliability of the proposed theoretical model, we also compared the ratio between maximal transversal trapping forces between each one of the three particles (PS, PMMA microsphere and yeast) and the glial structure obtained theoretically and experimentally, for left and right particle displacements in relation to the fiber. Results are shown in graphic 11. It is possible to conclude that theoretical results approximately match the experimental ones and that there is not a statistically significant difference between them $(\mathrm{p}>0.05$; Wilcoxon test, two-tailed). However, there is a discrepancy for the results obtained for the polystyrene particle. Probably, this model is not the most suitable to transduce trapping force dependence between the glial structure and the polystyrene microspheres. Further studies must be conducted to theoretically understand the trapping forces evolution with the nature of the particles. Probably, these models require the inclusion of more parameters about the scatterers, when they are complex and biologic.

\section{CONCLUSIONS}

Along this study, we provide an experimental characterization of the profile of trapping forces exerted by a polymeric lensed optical fiber on four different types of targets: a polystyrene and a PMMA microsphere, a yeast cell and a glial cellular structure found in a rodent primary culture of glial cells. Considering that the range of particles trapped and manipulated using fiber tweezers reported in literature is limited and that their mechanical effects on complex cells such as the neural-derived ones is poorly understood, our results could provide valuable insights to understand in which trapping conditions we can stably manipulate this kind of "demanding" structures. We have also tested the feasibility and reliability of a previously numerical model to characterize 


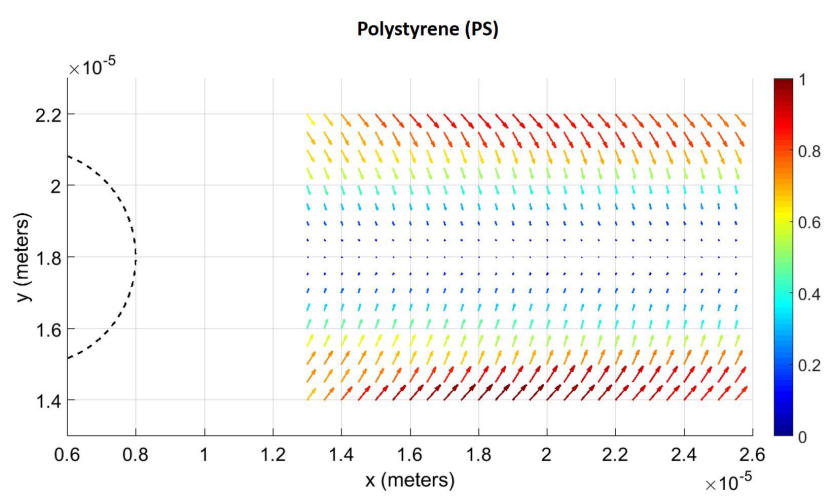

(A)

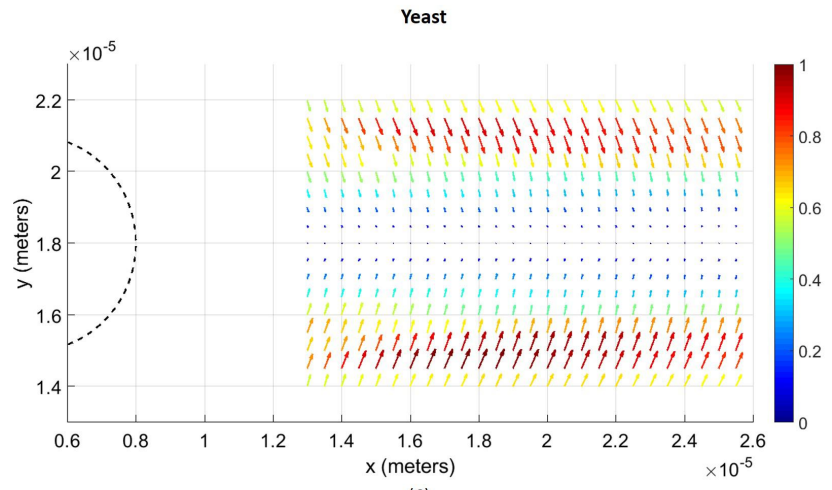

(c)

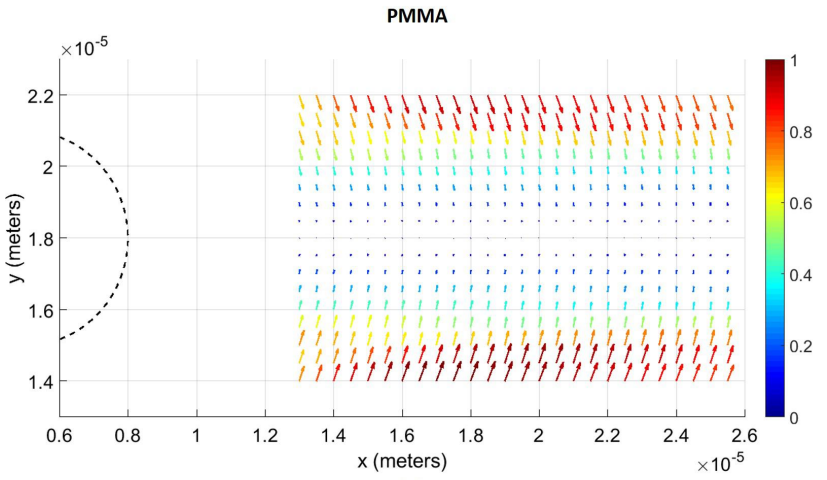

(B)

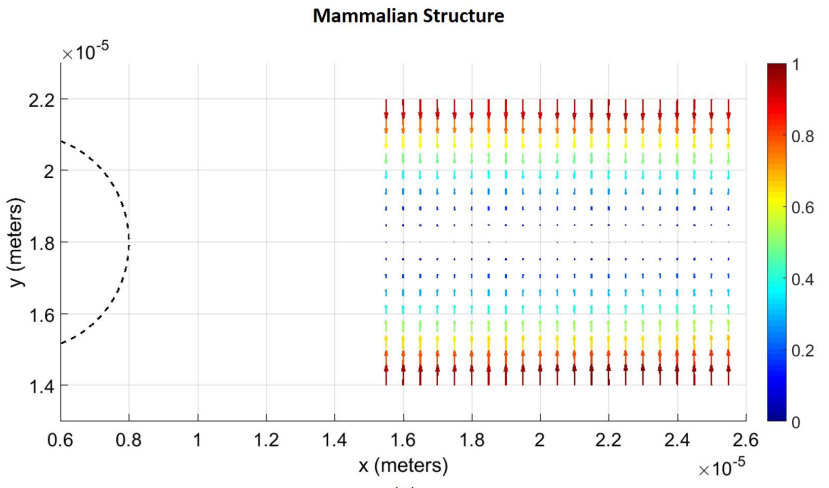

(D)

Figure 10. 2D trapping forces distribution maps for (A) PS particle, (B) PMMA particle, (C) yeast and (D) glial mammalian structure.

Transversal force vs. Refractive Index

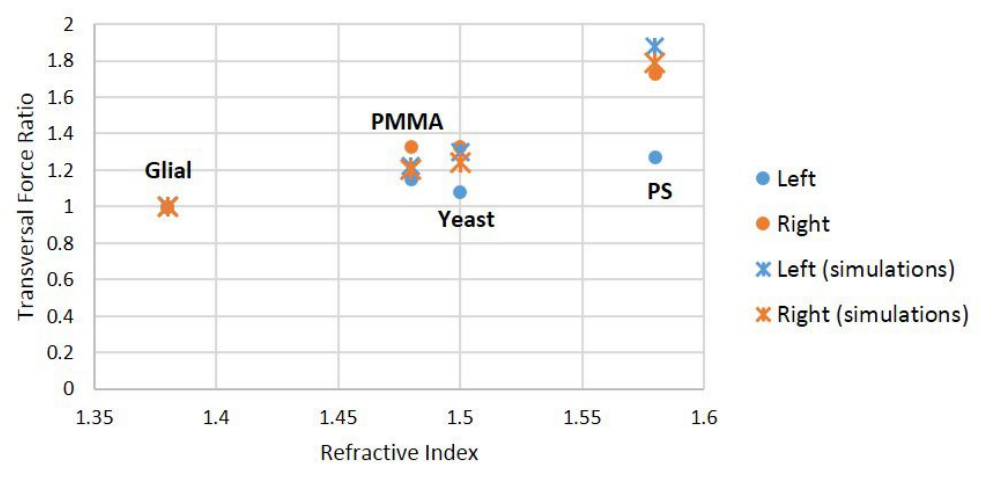

Figure 11. Ratio of maximal force magnitudes experimentally and theoretically obtained between the particles analyzed and the glial cell, for particle displacements to the left and to the right in relation to the propagation of the laser beam. Dots represent experimental results and crosses represent theoretical results.

trapping forces exerted by fiber tweezers considering this new scenario including complex targets. We found that theoretical calculations did not significantly differ from real ones. The models based on the Barnett and Loudon method $^{31}$ proposed so far are able to provide reliable results and probably can better math the real conditions after the inclusion of additional parameters or a more fine tuning of the ones already considered. 


\section{ACKNOWLEDGMENTS}

This work was partly developed under the project NanoSTIMA, funded by the North Portugal Regional Operational Program (NORTE 2020), under the PORTUGAL 2020 Partnership Agreement, and through the European Regional Development Fund (ERDF). It was also funded by the Portuguese Foundation for Science and Technology $(\mathrm{PD} / \mathrm{BD} / 135023 / 2017)$.

\section{REFERENCES}

[1] Paiva, J. S., Jorge, P. A. S., Rosa, C. C., and Cunha, J. P. S., "Optical fiber tips for biological applications: from light confinement, biosensing to bioparticles manipulation," Biochimica et Biophysica Acta (BBA) General Subjects, - (2018).

[2] Paiva, J. S., Ribeiro, R., Cunha, J. P. S., Rosa, C. C., and Jorge, P. A. S., "Single Particle Differentiation through 2D Optical Fiber Trapping and Back-scattered Signal Statistical Analysis: an Exploratory Approach," Sensors (2018).

[3] Ribeiro, R., Soppera, O., Oliva, A., Guerreiro, A., and Jorge, P. A. S., "New trends on optical fiber tweezers," Journal of Lightwave Technology 33(16), 3394-3405 (2015).

[4] Liberale, C., Di Fabrizio, E., Cojoc, G., Perozziello, G., Candeloro, P., Bragheri, F., Ferrara, L., Minzioni, P., and Cristiani, I., "Optical fiber tweezers fabricated by two photon lithography," in [The European Conference on Lasers and Electro-Optics], CLEB5_6, Optical Society of America (2011).

[5] Ashkin, A., "Acceleration and trapping of particles by radiation pressure," Physical Review Letters 24(4), 156 (1970).

[6] Maragò, O., Jones, P., Gucciardi, P., Volpe, G., and Ferrari, A., "Optical trapping and manipulation of nanostructures," Nature nanotechnology 8(11), 807-819 (2013).

[7] Ritchie, D. and Woodside, M., "Probing the structural dynamics of proteins and nucleic acids with optical tweezers," Current opinion in structural biology 34, 43-51 (2015).

[8] Paiva, J. S., Ribeiro, R., Jorge, P., Rosa, C., Guerreiro, A., and Cunha, J. P. S., "2D Computational Modeling of Optical Trapping Effects on Malaria-infected Red Blood Cells," in [Frontiers in Optics], JTu2A68, Optical Society of America (2017).

[9] Ribeiro, R., [Optical fiber tools for single cell trapping and manipulation], Doctoral thesis - University of Porto (2017).

[10] Paiva, J., Ribeiro, R., Jorge, P., Rosa, C., and Cunha, J., "Computational modeling of red blood cells trapping using Optical Fiber Tweezers," in [Bioengineering (ENBENG), 2017 IEEE 5th Portuguese Meeting on], 1-4, IEEE (2017).

[11] Liu, Y., Fiber Optical Tweezers For Microscale and Nanoscale Particle Manipulation and Force Sensing, $\mathrm{PhD}$ thesis (2011).

[12] Liu, X., Huang, J., Li, Y., Zhang, Y., and Li, B., "Rotation and deformation of human red blood cells with light from tapered fiber probes," Nanophotonics 6(1), 309-316 (2017).

[13] Barron, A., Kar, A., Aspray, T., Waddie, A., Taghizadeh, M., and Bookey, H., "Two dimensional interferometric optical trapping of multiple particles and escherichia coli bacterial cells using a lensed multicore fiber," Optics express 21(11), 13199-13207 (2013).

[14] Hao, F., Wang, R., and Wang, J., "A novel fiber optical tweezers with FZP structures on fiber end-face," in [International Conference on Optical Instrumentation and Technology], 75070K-75070K, International Society for Optics and Photonics (2009).

[15] Wang, T., Tang, X., Zhang, Y., Zhang, Y., and Liu, Z., "A novel single fiber optical tweezers based on GIMMF: simulation and experiment," in [25th International Conference on Optical Fiber Sensors], 103235A-103235A, International Society for Optics and Photonics (2017).

[16] Mohanty, S., Mohanty, K., and Berns, M., "Manipulation of mammalian cells using a single-fiber optical microbeam," Journal of biomedical optics 13(5), 054049-054049 (2008).

[17] Soppera, O., Jradi, S., and Lougnot, D., "Photopolymerization with microscale resolution: Influence of the physico-chemical and photonic parameters," Journal of Polymer Science Part A: Polymer Chemistry 46(11), 3783-3794 (2008). 
[18] Ribeiro, R. R., Soppera, O., Guerreiro, A., and Jorge, P., "Polymeric optical fiber tweezers as a tool for single cell micro manipulation and sensing," in [International Conference on Optical Fibre Sensors (OFS24)], 96342K-96342K, International Society for Optics and Photonics (2015).

[19] Ribeiro, R., Jorge, P., and Guerreiro, A., "New developments on the design and modeling of fiber optical tweezers," in [8th Ibero American Optics Meeting/11th Latin American Meeting on Optics, Lasers, and Applications], International Society for Optics and Photonics (2013).

[20] Ribeiro, R., Queirós, R., Guerreiro, A., Ecoffet, C., Soppera, O., and Jorge, P., "Fiber optical beam shaping using polymeric structures," in [OFS2014 23rd International Conference on Optical Fiber Sensors], 91573K91573K, International Society for Optics and Photonics (2014).

[21] Ribeiro, R., Queirós, R., Ecoffet, C., Soppera, O., Oliva, A., Guerreiro, A., and Jorge, P., "Rapid fabrication of polymeric micro lenses for optical fiber trapping and beam shaping," in [SPIE NanoScience+ Engineering], 91642M-91642M, International Society for Optics and Photonics (2014).

[22] Neuman, K. and Block, S., "Optical trapping," Review of scientific instruments 75(9), 2787-2809 (2004).

[23] Sultanova, N., Kasarova, S., and Nikolov, I., "Dispersion properties of optical polymers," Acta Physica Polonica-Series A General Physics 116(4), 585 (2009).

[24] Sigrist-Photometer, A., [Refractive Index.] (2017).

[25] Bereiter-Hahn, J., Fox, C., and Thorell, B., "Quantitative reflection contrast microscopy of living cells.," The Journal of cell biology 82(3), 767-779 (1979).

[26] Paul, J. and Mateyko, G., "Quantitative interference microscopy of polytene chromosomes: I. cytophysical studies on refractive index and dry mass concentration," Experimental cell research 59(2), 227-236 (1970).

[27] Svoboda, K. and Block, S., "Biological applications of optical forces," Annual review of biophysics and biomolecular structure 23(1), 247-285 (1994).

[28] Piccinini, F., Kiss, A., and Horvath, P., "CellTracker (not only) for dummies," Bioinformatics 32(6), 955$957(2015)$.

[29] Coffey, W. and Kalmykov, Y., [The Langevin equation: with applications to stochastic problems in physics, chemistry and electrical engineering], World Scientific (2004).

[30] Paiva, J. S., Ribeiro, R., Jorge, P., Rosa, C., and Cunha, J. P. S., "Computational modeling of red blood cells trapping using optical fiber tweezers," in [2017 IEEE 5th Portuguese Meeting on Bioengineering (EN$B E N G)], 1-4$ (Feb 2017).

[31] Barnett, S. and Loudon, R., "On the electromagnetic force on a dielectric medium," Journal of Physics B: Atomic, Molecular and Optical Physics 39(15), S671 (2006).

[32] Oskooi, A., Roundy, D., and Ibanescu et al., M., "MEEP: A flexible free-software package for electromagnetic simulations by the FDTD method," Computer Physics Communications 181(3), 687-702 (2010).

[33] Rodrigues Ribeiro, R. S., Guerreiro, A., Ecoffet, C., Soppera, O., and Jorge, P. A. S., "New theoretical and experimental methods for the design of fiber optic tweezers," in [Proc. SPIE 8794, Fifth European Workshop on Optical Fibre Sensors], Proc. SPIE 8794, 87941P-87941P-4 (2013). 